\title{
Application de la cryométrie à la mesure de l'hydrolyse enzymatique du lactose
}

\author{
par \\ J. P. RAMET*, G. NOVAK*, P.A. EVERS** \\ et H. H. NIJPELS***
}

\section{INTRODUCTION}

Depuis quelques années, l'utilisation de la $\beta$-galactosidase est préconisée pour résoudre les problèmes d'intolérance au lactose présentés par certaines populations et individus $[1,4]$, pour fabriquer certains produits laitiers fermentés $[5,7,9,11,14,15]$ et pour élaborer des lactosérums concentrés hydrolysés $[8,12]$.

Il existe différentes méthodes pour contrôler l'hydrolyse du lactose ; les plus connues font appel à des méthodes classiques ou plus élaborées comme la chromatographie en phase gazeuse $[8,10]$ et les méthodes de dosage enzymatique $[2,3,6]$ mais toutes sont relativement longues, délicates et nécessitent parfois un équipement sophistiqué, les laboratoires industriels ne disposent pas toujours des équipements nécessaires. De plus, certaines méthodes donnent des résultats difficilement reproductibles quantitativement [7].

Nous proposons ci-après, une méthode rapide, nouvelle, basée sur la mesure de l'abaiss əment cryoscopique du point de congélation de la solution, consécutif à l'hydrolyse du lactose.

\footnotetext{
* Ecole Nationale Supérieure Agronomique et des Industries Alimentaires, Nancy (France).

** Campina, Rykevoort (Pays-Bas).

*** Gist-Brocades, Delft (Pays-Bas).
} 


\section{MATERIELS ET METHODES}

\section{II.1. Principe de la méthode}

Lors de l'hydrolyse du lactose par voie enzymatique, la molécule de lactose (PM 342) est scindée en deux oses simples, glucose et galactose (PM 180) selon le schéma bien connu suivant :

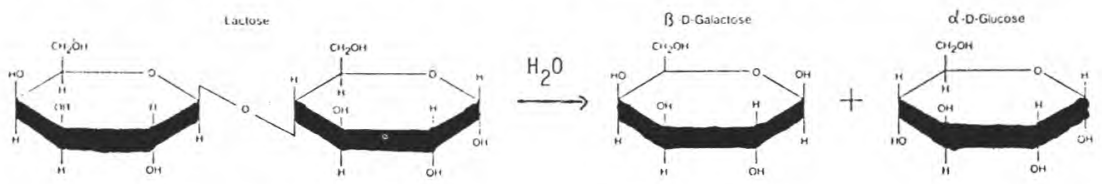

Cette hydrolyse s'accompagne d'un abaissement corrélatif du point de congélation de la solution, défini par la relation :

$$
\Delta \mathrm{T}=-\frac{\mathrm{X}}{\mathrm{M}} \mathrm{K}
$$

ou $\mathrm{X}=$ concentration de la substance (non ionique) en solution aqueuse $(\mathrm{g} / \mathrm{l})$;

$\mathrm{M}=$ poids moléculaire de la substance ;

$\mathrm{K}=$ constante cryoscopique de l'eau : $\mathrm{K}=1,86$.

Ainsi, pour une solution aqueuse à $50 \mathrm{~g} / \mathrm{l}$ de lactose dans l'eau, le point de congélation sera :

$$
\Delta \mathrm{T}_{1}=\frac{50}{342} \times 1,86=-0,272^{\circ} \mathrm{C}
$$

Si ce lactose est totalement hydrolysé en glucose et galactose, le point de congélation sera, compte tenu de la correction apportée en raison de l'eau fixée :

$$
\Delta \mathrm{T}_{2}=\frac{52,6}{1,80} \times \frac{100}{99,74} \times 1,86=-0,545^{\circ} \mathrm{C}
$$

L'hydrolyse complète du lactose en solution à $50 \mathrm{~g} / 1$ entraînera donc un abaissement cryoscopique du point de congélation de $-0,273^{\circ} \mathrm{C}$.

De ce fait, le point de congélation d'un lait serait porté après hydrolyse de $-0,540^{\circ} \mathrm{C}$ à $-0,813^{\circ} \mathrm{C}$.

De la même façon, lors d'une hydrolyse enzymatique du lactose, l'abaissement cryoscopique devrait refléter le taux d'hydrolyse.

\section{II.2. Méthodes}

Pour vérifier les hypothèses précédentes, nous avons mesuré sur deux substrats lactosés différents (lait - ultrafiltrat) la libération du 
galactose et du glucose et établi la corrélation entre la méthode cryoscopique proposée et deux méthodes de dosage enzymatique $[2,6]$.

\section{iII.2.1. Préparation de la $\beta$-galactosidase}

La $\beta$-galactosidase utilisée pour l'hydrolyse du substrat provient d'une levure Kluyveromyces lactis, elle est commercialisée par GistBrocades N.V. sous la dénomination Maxilact [8].

La préparation enzymatique possède une activité $\beta$-galactosidasique de 30000 O.N.P.G. soit 7500 N.L.U. [8] et a été utilisée en solution aqueuse de 1 à 4 p. 100 selon les essais.

\section{II.2.2. SUBSTRAT}

Le lait est un lait écrémé reconstitué à partir de poudre "lowheat " de bonne qualité bactériologique et d'eau stérile; la teneur en lactose est de $42 \mathrm{~g} / 1$ à $50 \mathrm{~g} / 1$ selon les essais.

L'ultrafiltrat utilisé provient d'un lait reconstitué dans les conditions précitées et ultrafiltré sur membrane $\mathrm{P} 30$ à l'aide d'un module Diaflo ; sa teneur en lactose est de $44 \mathrm{~g} / 1$.

Les milieux ont été préchauffés à 30 ou $35^{\circ} \mathrm{C}$ selon les essais, puis répartis en flacons de $150 \mathrm{ml}$ placés au bain-marie thermostaté

\section{III.2.3. CONDITIONS D'HYDROLYSE}

La préparation enzymatique de $\beta$-galactosidase a été ajoutée dans le substrat à une concentration de 1500 à 6000 N.L.U./1 selon les essais.

La température choisie a été maintenue pendant toute la durée de l'hydrolyse.

\section{II.2.4. Mesure de LA CinéTIQue d'Hydrolyse}

Deux types de méthodes ont été comparées :

- Méthode enzymatique

L'hydrolyse a été suivie d'une part :

- par la mesure du galactose formé ; ce dernier a été oxydé en présence de galactose déshydrogénase et le NAD réduit correspondant a été mesuré à $340 \mathrm{~nm} \mathrm{[2]} \mathrm{;}$

- par la mesure du glucose formé ; ce dernier a été oxydé en présence de glucose oxydase ; le peroxyde d'hydrogène libéré au cours de la réaction contribue à la formation d'un composé coloré dont la concentration est mesurée par spectrophotométrie à $510 \mathrm{~nm}$ [6].

\section{- Méthode cryoscopique}

La cinétique de l'hydrolyse a été suivie en mesurant l'abaissement cryoscopique du point de congélation à l'aide d'un cryoscope Advanced-Instruments type Hi-precision Research Osmometer 3 R. 
Les analyses ont été faites simultanément par l'une ou l'autre des méthodes enzymatiques et la méthode cryoscopique à to, puis toute les $15 \mathrm{mn}$ pendant $1 \mathrm{~h} 30$ à $3 \mathrm{~h}$ selon les essais.

\section{RESULTATS - DISCUSSION}

\section{III.1. Corrélation entre méthode cryoscopique et méthode enzymatique}

La méthode cryoscopique permet de suivre la cinétique d'hydrolyse de façon très régulière dans le lait et l'ultrafiltrat (fig. 1, tab. 1).

\section{TABLEAU 1}

\begin{tabular}{|c|c|c|c|c|c|c|c|c|c|c|}
\hline \multirow{2}{*}{$\begin{array}{c}\text { Temps } \\
\text { incubation } \\
\text { (h) }\end{array}$} & \multicolumn{2}{|c|}{$\begin{array}{c}\text { Essai } 1 \\
\text { hydrolyse } \\
\text { p. } 100\end{array}$} & \multicolumn{2}{|c|}{$\begin{array}{c}\text { Essai } 2 \\
\text { hydrolyse } \\
\text { p. } 100\end{array}$} & \multicolumn{2}{|c|}{$\begin{array}{c}\text { Essai } 3 \\
\text { hydrolyse } \\
\text { p. } 100\end{array}$} & \multicolumn{2}{|c|}{$\begin{array}{c}\text { Essai } 4 \\
\text { hydrolyse } \\
\text { p. } 100\end{array}$} & \multicolumn{2}{|c|}{$\begin{array}{c}\text { Essai } 5 \\
\text { hydrolyse } \\
\text { p. } 100\end{array}$} \\
\hline & MC & $\mathrm{ME}$ & $M C$ & $\mathrm{ME}$ & MC & ME & MC & $\mathrm{ME}$ & MC & $\mathrm{ME}$ \\
\hline 0 & 0 & 0 & 0 & 0 & 0 & 0 & 0 & 0 & 0 & 0 \\
\hline 1 & 17 & 18 & 17 & 17 & 20 & 18 & 17 & 18 & 18 & 17 \\
\hline 2 & 29 & 31 & 28 & 30 & 30 & 31 & 28 & 29 & 29 & 29 \\
\hline 3 & 43 & 40 & 41 & 40 & 42 & 41 & 38 & 39 & 40 & 38 \\
\hline 4 & 50 & 51 & 49 & 49 & 51 & 49 & 47 & 48 & 49 & 48 \\
\hline
\end{tabular}

Comparaison du pourcentage d'hydrolyse du lactose déterminé par la méthode cryoscopique (MC) et la méthode enzymatique à la glucoeoxydase (ME.).

Conditions de l'hydrolyse : substrat : lait écrémé reconstitué ; lactose : $50 \mathrm{~g} / 1$; température : $30^{\circ} \mathrm{C}$; Maxilact : 500 N.L.U./1.

La droite de régression entre la méthode enzymatique et la méthode cryoscopique présente une très bonne corrélation pour les deux milieux étudiés (fig. 2 et 3 ) ; les valeurs de F montrent que cette corrélation est hautement significative.

La similitude des équations des droites pour le lait et l'ultrafiltrat montre qu'il n'y a pas d'interférence des constituants des milieux lors des mesures et que la variation du point de congélation est seule fonction de l'hydrolyse du lactose. 


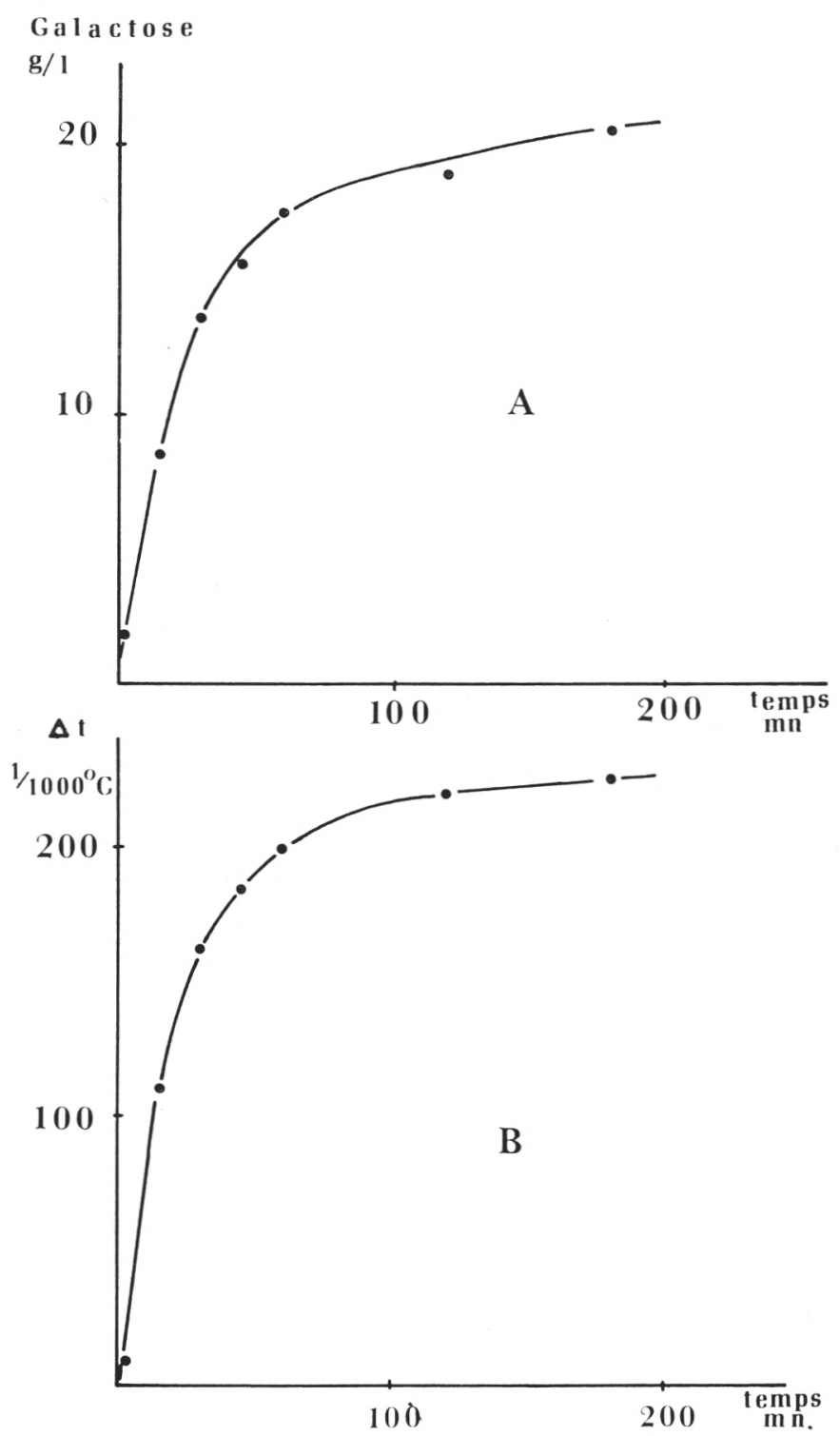

fig. 1

Cinétique d'hydrolyse du lactose dans le lait :

A : mesurée par la méthode enzymatique.

B : mesurée par la méthode cryoscopique.

(Conditions de l'hydrolyse : substrat : lait écrémé reconsti-

tué ; lactose : $44 \mathrm{~g} / 1$; température : $35^{\circ} \mathrm{C}$; Maxilact :

3000 N.L.U./l). 


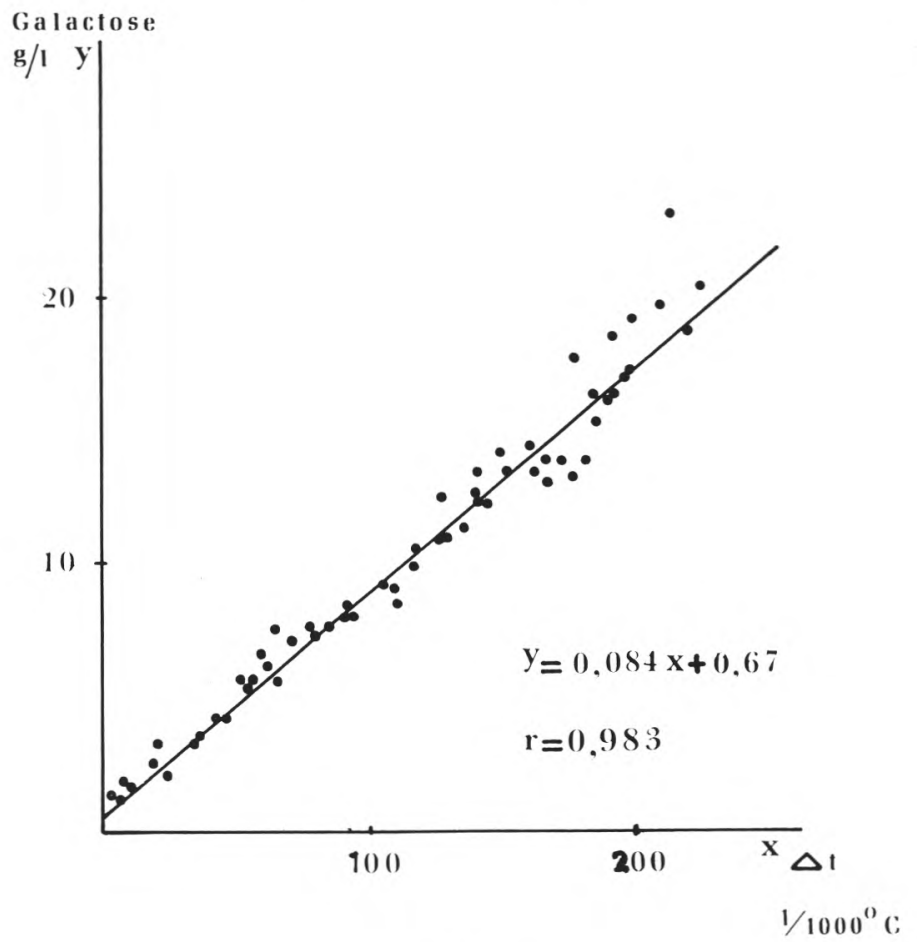

fig. 2

Mesure de l'hydrolyse dans le lait : corrélation entre la méthode cryoscopique et la méthode enzymatique (galactosedéshydrogénase).

Equation de la droite :$$
\mathrm{y}=0,084+0,67
$$

Coefficient de corrélation :

$\mathrm{r}=0,983$

Nombre de couples de mesures : $n=63$

$$
\mathrm{F}=1749
$$

Après hydrolyse totale du lactose du lait (lactose $50 \mathrm{~g} / 1$; enzyme 5000 N.L.U./1, 35 $\mathrm{C}, 4 \mathrm{~h}$ ), l'abaissement du point de congélation mesuré est de $-0,274^{\circ} \mathrm{C}$ par rapport au lait témoin, cette valeur correspond étroitement à la valeur théorique calculée $-0,272^{\circ} \mathrm{C}$.

\section{III.2. Précision}

Compte tenu de la précision du cryoscope utilisé $\left(5 / 10000^{\circ} \mathrm{C}\right)$, l'erreur maxima relative sur la mesure du point de congélation est de l'ordre de $1 / 1000$; cette erreur est donc négligeable. 


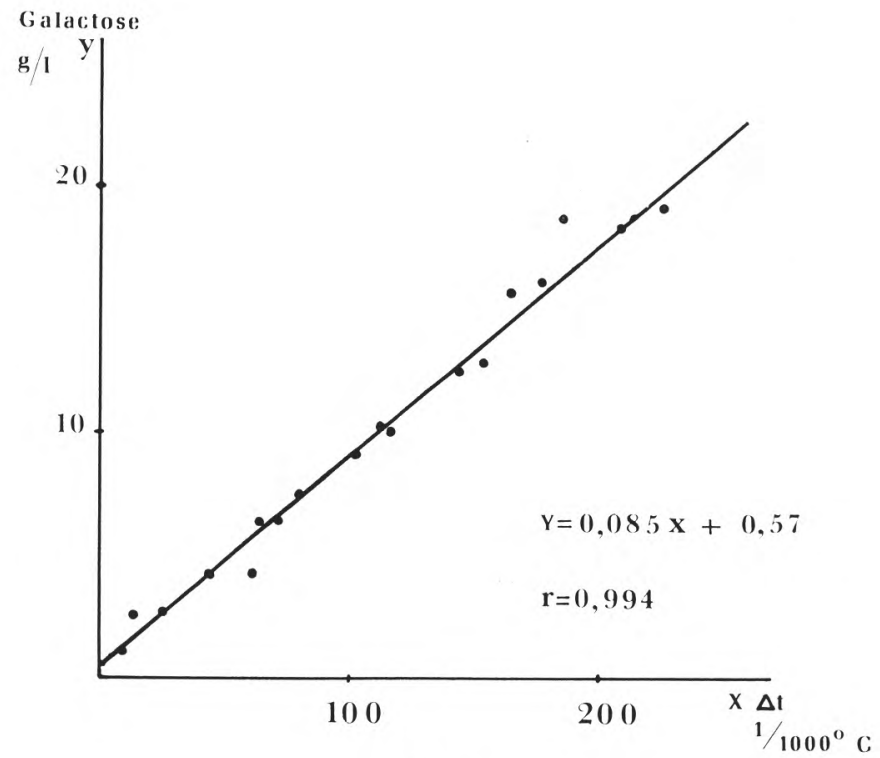

fig. 3

Mesure de l'hydrolyse du lactose dans l'ultrafiltrat de lait: corrélation entre la méthode cryoscopique et la méthode enzymatique (galactosedéshydrogénase).

Equation de la droite : $\quad \mathrm{y}=0,085+0,57$

Coefficient de corrélation : $\quad \mathrm{r}=0,994$

Nombre de couples de mesures : $\mathrm{n}=19$

$\mathrm{F}=1404$

TABLEAU 2

\begin{tabular}{c|c|c|c}
\hline $\begin{array}{c}\text { Nombre de } \\
\text { mesures }\end{array}$ & Moyenne & Ecart-type & $\begin{array}{c}\text { Coefficient } \\
\text { variation }\end{array}$ \\
\hline $\mathbf{M}=10$ & $\overline{\mathrm{M}}=-0,7930^{\circ} \mathrm{C}$ & $\Delta=0,0034$ & $\mathrm{~V}=0,43$ p. 100 \\
\hline
\end{tabular}

Caractéristiques de répétabilité de la méthode cryoscopique.

Condition de l'hydrolyse : substrat : lait écrémé reconstitué ; lactose : $44 \mathrm{~g} / 1$; température : $35^{\circ} \mathrm{C}$; Maxilact : 4500 N.L.U./1 ; durée : $3 \mathrm{~h}$. 


\section{III.3. Répétabilité}

Sur une série de 10 mesures du point de congélation d'un même échantillon mesuré après l'hydrolyse totale du lactose (tab. 2), le coefficient de variation est de 0,43 p. 100 , la répétabilité de la méthode proposée peut donc être considérée comme très satisfaisante.

\section{CONCLUSION}

La méthode cryoscopique proposée pour contrôler l'hydrolyse du lactose est remarquable par sa simplicité ; elle possède une bonne répétabilité et une bonne précision.

Pratiquement les équations de corrélation que nous avons établies permettent de calculer directement le taux d'hydrolyse du lait ou d'un ultrafiltrat. Il suffit de déterminer pour cela l'abaissement du point de congélation du milieu par rapport à une valeur témoin correspondant au milieu non hydrolysé.

Il est également possible d'établir en raison de la relation linéaire entre l'abaissement de température et le taux d'hydrolyse, une droite étalon passant par l'origine et le point dont les coordonnées correspondent à une hydrolyse de 100 p. 100. L'abaque ainsi établie permet de déduire facilement pour chaque valeur de $\Delta t$ mesurée le taux d'hydrolyse correspondant. Il faut toutefois remarquer que cette deuxième méthode est approchée, la droite ne passant pas rigoureusement par l'origine. L'erreur commise est toutefois négligeable $(0,10$ p. 100$)$.

L'intérêt pratique essentiel de cette méthode est sa rapidité, la mesure du point de congélation se faisant en moins de $5 \mathrm{mn}$; de plus beaucoup de laboratoires laitiers étant actuellement équipés de cryoscopes, elle ne demande aucun investissement supplémentaire en matériels et réactifs. Le coût de l'analyse est donc faible.

Pour ces différentes raisons, cette méthode est très intéressante à la fois pour contrôler l'hydrolyse du lactose et tester l'activité de préparations $\beta$-galactosidasiques.

Il convient toutefois de souligner que les techniques de mesures par cryoscopie manquent de spécificité et que les résultats sont erronés chaque fois que la composition du milieu en substances solubles varie, en particulier dans le cas d'acidification ou d'addition de sels auxiliaires de fabrication. La méthode proposée n'est rigoureuse qu'en application à des milieux microbiologiquement et chimiquement stables. 


\section{Ré s u m é}

L'hydrolyse du lactose entraîne un abaissement du point de congélation du lait et de l'ultrafiltrat.

Une corrélation linéaire existe entre l'abaissement cryoscopique et la quantité de galactose libéré. L'équation de la droite de régression $\mathrm{y}=0,084 \mathrm{X}+0,67$ ( $\mathrm{Y}$ est la teneur en galactose $\mathrm{g} / \mathrm{l}$ et $\mathrm{X}$ l'abaissement cryoscopique dû à l'hydrolyse du lactose en $10^{-3}{ }^{\circ} \mathrm{C}$ ) permet de connaître le taux d'hydrolyse du lait.

Cette nouvelle méthode rapide et précise peut être utilisée avantageusement au laboratoire et en usine. Une correction des résultats est nécessaire chaque fois que la composition du milieu en substances solubles autres que les produits d'hydrolyse varie.

\section{S u m m a ry}

The lactose's hydrolysis involves a lowering of freezing point of milk and ultrafiltrate. A linear correlation has been finded between freezing point's depress and produced galactose's. The equation of regression line $\mathrm{Y}=0,084 \mathrm{X}+0,67$ ( $\mathrm{Y}=$ galactose's content in $\mathrm{g} / 1 \mathrm{X}=$ freezing point's depress by lactose's hydrolysis in $10^{-3}{ }^{\circ} \mathrm{C}$ ) makes easy determination of hydrolysis level.

The new method, rapid and precise is well adapted for dairy laboratories and plant's controls.

The results must be corrected every time some changing in soluble substances othes than hydrolysis products occurs.

Reçu pour publication en décembre 1978.

\section{Bibliographie}

[1] Beaufrand (M. J.), Poullain (B.), Munsch (B.) et Debry (G.) (1957). - Comparaison chez l'homme de l'absorption du lactose et du lactose hydrolysé. Le Lait, 57, 568, 501-508.

[2] Bofhringer - Mannheim (1977). - Doc. méthodes d'analyse enzymatique en chimie alimentaire.

[3] Cheng (F. S.) and Christian (G. D.) (1977). - Rapid enzymatic determination of lactose in food products using amperometric measurement of the rate of depletion of oxygen. Analyst 102, 124-131.

[4] Davis (A. E.) and Bolin (T. D.) (1967). - Lactose Intolerance in Asians. Nature, 216, 1244-1245.

[5] Gyuricsek (D. M.) and Thompson (M. P.) (1976). - Hydrolyzed lactose cultured dairy products. Cultured Dairy Products, August, 12-13. 
[6] Merck - DarmstadT. - Détermination photométrique de la concentration en glucose. Documentation technique.

[7] Mouillet (L.), Luquet (E M.) et Boudier (J. F.) (1977). — Détermination des sucres par chromatographie en phase gazeuse. Le Lait, 57, 561-562, 37-54.

[8] NiJpels (H. H.) (1977). - Maxilact Lactase in dairy industry. North European Dairy J., 10, 358-360, 11-12, 382-385.

[9] Olling (G. J.) (1972). - Lactase treatment in dairy industry. An. Technol. Agric., 21, 3, 343-356.

[10] Pastuska (G.) (1961). - Untersuchungen über die qualitative und quantitative Bestimmung der Zucker mit Hilfe Kieselsgeschicht Chromatographie. Ztg anl. Chem., 179, 427-429.

[11] Ramet (J. P.) (1978). - Applications de l'hydrolyse du lactose en fromagerie. Communication. Journée APRIA, 25 avril 1978, 35, rue du Général-Foy 75008 Paris.

[12] Roger (L.), Thapon (J. L.), Maubois (J. L.) et Brulé (G.) (1976). - Hydrolyse $\mathrm{du}$ lactose contenu dans l'ultrafiltrat de lait ou de lactosérum en réacteur enzymatique à membrane. Le Lait, 56, 551-552, 56-75.

[13] Siegenthaler (U.) und Ritter (W.) (1977). - Eine Rasche DC. Methode zum nachweis von Mono und Dissachariden in Milchmischgetränken un Yoghurt. Mitt. Gebiete Lebensm. Hyg., 68, 448-450.

[14] Tamine (A. Y.) (1977). - The Behaviour of different starters cultures during the manufacture of yoghourt from hydrolyzed milk. Dairy Ind. Int., 42, 8, 7-11.

[15] Thompson (M. P.) and Brower (D. P.) (1974). - Manufacture of Cheddar cheese from hydrolyzed lactase milk. J. Dairy Sci., 57, 598. 